\title{
HENSELIAN VALUED FIELDS WITH PRESCRIBED VALUE GROUP AND RESIDUE FIELD
}

BY

LINDA HILL

\begin{abstract}
A class of fields supplementary to the inertia field of a given henselian valued field is used to construct extensions of that field having prescribed value group and residue field. The extensions so-constructed are characterized, and their number investigated.
\end{abstract}

1. Introduction. The construction of valued fields extending a given one and having prescribed value groups and residue fields with respect to the extensions of the given valuation has received much attention in the literature from many points of view; see [1], [2], [3], [5], [6], [7], [8], [11], [12], for example. The general problem, that of finding an extension $L$ of a given Krull-valued field $(K, A)$ such that the extensions of $A$ to $L$ realize prescribed value groups and residue fields, or, more generally, realizing simultaneously such prescriptions for the extensions of an entire tree of valuation rings of $K$, has yielded to a method initiated by Endler in [3] and Chapter IV of [4], for rank 1 Krull valuations and extended by the present author in [6] to a class of Krull valuations of finite rank. That is the realization of each prescribed value group and residue field extension by an extension of the henselization (completion, in the rank 1 case) of the given valued field, coupled with the realization of those fields as the henselizations of some single extension $L$ of $K$. Since a henselization of any valued field is an immediate extension of that field, the realization of prescribed value group and residue field extensions is achieved. Thus, the existence of extensions of a given henselian valued field having prescribed value group and residue field is of much interest. This paper presents a method for the construction of such fields and offers characterizations of the fields so-constructed. Offered also is a discussion of the number of extensions realizing a given prescription; some open questions on the subject are listed.

2. Preliminaries and notation. Throughout this work, if $A$ and $B$ are any pair of groups, rings, or fields, with $A \subseteq B$, we will denote by $\{B \mid A\}$ the lattice

Received by the editors August 17, 1973.

AMS (MOS) subject classifications (1970). Primary 12J10, 12B10; Secondary 12F10, $10 \mathrm{M} 10,13 \mathrm{~J} 15$.

Key words and phrases. Krull valuation, hen selian valued field, decomposition field, inertia field, ramification field. 
(under products, intersections, and set-theoretic inclusion) of subgroups, subrings, or subfields of $B$ containing $A$.

Let $(K, A)$ be a (Krull-) valued field, $\Omega$ a separable closure of $K$, and $C$ an extension of $A$ to $\Omega$. We will denote by $\zeta_{C}$ the place of $\Omega$ determined by $C$ and will write; for any $L \in\{\Omega \mid K\}, \zeta_{C} L$ for the residue field $\zeta_{C \cap L}(L)$; we write $\Gamma_{C}{ }^{L}$ for the value group $v_{C} \cap_{L}(L)$. Within the extension $\Omega \mid K$ one finds the wellknown intermediate fields $K^{Z}(C), K^{T}(C)$, and $K^{V}(C)$, the decomposition field, inertia field, and ramification field, respectively, of $C$ over $K$.

The fundamental properties of these fields which will be used in the present work are:

(2.1) $\left(K^{Z}(C), C \cap K^{Z}(C)\right)$ is a henselian valued field, minimal in $\{\Omega \mid K\}$ with respect to that property, and is an immediate extension of $(K, A)$. The field $(K, A)$ is henselian exactly in case $K^{Z}(C)=K$.

(2.2) $\left(K^{T}(C), C \cap K^{T}(C)\right)$ is an unramified extension of $(K, A)$ and is the unique maximal member of $\left\{\Omega \mid K^{Z}(C)\right\}$ with respect to that property; it is normal over $K^{Z}(C)$. Thus $\Gamma_{C}\left(K^{T}(C)\right)=\Gamma_{C} K ; \zeta_{C}\left(K^{T}(C)\right)=\zeta_{C} \Omega$, an algebraic closure of $\zeta_{A} K$. The place $\zeta_{C}$ is an order-preserving, degree-preserving lattice-isomorphism from $\left\{K^{T}(C) \mid K^{Z}(C)\right\}$ onto $\left\{\zeta_{C} \Omega \mid \zeta_{A} K\right\}$. The Galois groups Aut $\left(K^{T}(C) \mid K^{Z}(C)\right)$ and $A u t\left(\zeta_{C} \Omega \mid \zeta_{A} K\right)$ are isomorphic. For any member $L$ of $\left\{K^{V}(C) \mid K^{Z}(C)\right\}, L \cap$ $K^{T}(C)$ is the unique member of $\left\{K^{T}(C) \mid K^{Z}(C)\right\}$ having residue field $\zeta_{C} L_{\text {. }}$

(2.3) $\left(K^{V}(C), C \cap K^{V}(C)\right)$ is a tamely ramified extension of $(K, A)$ and contains every finite defectless (see [4]) tamely ramified extension $(L, C \cap L)$ of $(K, A)$ within $(\Omega, C)$; it is normal over both $K^{T}(C)$ and $K^{Z}(C)$. The valuation $v_{C}$ is an order-preserving, degree-preserving lattice-isomor phism from $\left\{K^{V}(C) \mid K^{T}(C)\right\}$ to $\left\{\Gamma\left(K^{V}(C)\right) \mid \Gamma_{A} K\right\}$. For any $L \in\left\{K^{V}(C) \mid K^{T}(C)\right\}, L \cdot K^{T}(C)$ is the unique member of $\left\{K^{V}(C) \mid K^{T}(C)\right\}$ having value group $\Gamma_{C} L$.

If the extension $C$ of $A$ to $\Omega$ is considered fixed, we will denote $K^{Z}(C)$, $K^{T}(C)$, and $K^{V}(C)$ by $K^{Z}, K^{T}$, and $K^{V}$, respectively, and will omit reference to $C$ in $\Gamma_{C}, v_{C}$, and $\zeta_{C}$

3. Neukirch complements. In his paper [10], Jürgen Neukirch established the existence of fields which we have chosen to call Neukirch complements, supplementary to the three fields discussed in $\$ 2$ above. Namely, Neukirch showed that the exact sequence

(3.1) $\{1\} \rightarrow$ Aut $\left(K^{V} \mid K^{T}\right) \rightarrow$ Aut $\left(K^{V} \mid K^{Z}\right) \stackrel{j}{\rightarrow}$ Aut $\left(K^{V} \mid K^{Z}\right) /$ Aut $\left(K^{V} \mid K^{Z}\right) \rightarrow\{1\}$

splits. A splitting of sequence (3.1) is obtained if a field $K^{S} \in\left\{K^{V} \mid K^{Z}\right\}$ is found for which $K^{S} \cdot K^{T}=K^{V}$ and $K^{S} \cap K^{T}=K^{Z}$; Neukirch showed that the fields $K^{S}$ with those properties are precisely the members of $\left\{K^{V} \mid K^{T}\right\}$ maximal 
with respect to the property $K^{S} \cap K^{T}=K^{Z}$ (that is, that $\zeta K^{S}=\zeta K$ ); a simple Zorn's lemma argument thus establishes the existence of such fields. We have called (see [6]) such a field $K^{S}$ a Neukirch complement for $K^{T}$ in $K^{V}$.

The main property of Neukirch complements which Neukirch establishes is:

Theorem (3.2) (Neukirch). The lattices $\left\{K^{S} \mid K^{Z}\right\}$ and $\left\{\Gamma K^{S} \mid \Gamma K^{Z}\right\}=\left\{\Gamma K^{V} \mid \Gamma K\right\}$ are in order-preserving, degree-preserving correspondence under the lattice-isomorphisms $L \mapsto \Gamma L$ and $V^{-1}[\Delta] \mapsto \Delta$. The composition of this isomorphism with that from $\left\{\Gamma K^{V} \mid \Gamma K\right\}$ onto $\left\{K^{V} \mid K^{T}\right\}$ preserves value groups. That composition is $L \mapsto L \cdot K^{T}$ with inverse $M \cap K^{S} \leftrightarrow M$.

4. Neukirch-determinable extensions. Here and in the sequel we assume $(K, A)$ is henselian, so $K^{Z}=K$. If $L$ is any member of $\left\{K^{V} \mid K\right\}$, denote by $L^{T}$ the inertia field $L^{T}(C)=L \cdot K^{T}(C)$ of $C$ over $L$. The ramification field of $C$ over $L$ is $K^{V}$. The group $\operatorname{Aut}\left(K^{V} \mid L\right)$ is a subgroup of $\operatorname{Aut}\left(K^{V} \mid K\right), \operatorname{Aut}\left(K^{V} \mid L^{T}\right)$ is a subgroup of $\operatorname{Aut}\left(K^{V} \mid K^{T}\right)$, and $\operatorname{Aut}\left(K^{V} \mid K^{T}\right) \cap \operatorname{Aut}\left(K^{V} \mid L\right)=\operatorname{Aut}\left(K^{V} \mid L^{T}\right)$, so the following diagram commutes:

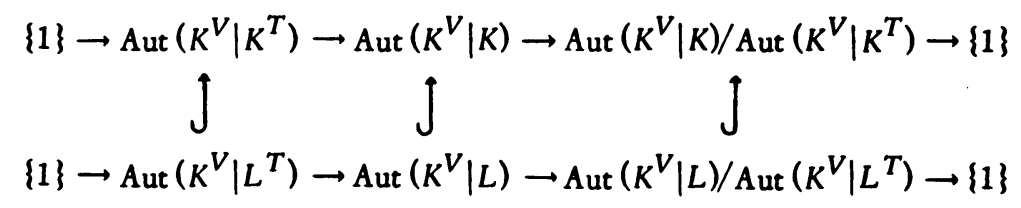

If $\eta: \operatorname{Aut}\left(K^{V} \mid K\right) / \operatorname{Aut}\left(K^{V} \mid K^{T}\right) \rightarrow \operatorname{Aut}\left(K^{V} \mid K\right)$ is a splitting of sequence (4.1), then the restriction of $\eta$ to $\operatorname{Aut}\left(K^{V} \mid L\right) / \operatorname{Aut}\left(K^{V} \mid L^{T}\right)$ splits sequence (4.2) in such a way that the resulting diagram commutes, if and only if $\eta\left(\operatorname{Aut}\left(K^{V} \mid L\right) / \operatorname{Aut}\left(K^{V} \mid L^{T}\right)\right) \subseteq$ $\operatorname{Aut}\left(K^{V} \mid L\right)$. In case this happens, we have called $L$ a Neukirch-determinable extension of $K$. If $\operatorname{Im}(\eta)=K^{S}$, we also say that $L$ is a $K^{S}$-determinable extension of $K$. Clearly, then, $L$ is $K^{S}$-determinable if and only if $L \cdot K^{S}$ is a Neukirch complement for $L^{T}$ in $K^{V}$.

Proposition (4.3). If $L \in\left\{K^{V} \mid K\right\}$ and $K^{S}$ is a Neukircb complement for $K^{T}$ in $K^{V}$, then $L$ is $K^{S}$-determinable if and only if $L \cdot K^{S} \cap L \cdot K^{T}=L$. Further, for any member $L$ of $\left\{K^{V} \mid K\right\}$, the field $L \cdot K^{S} \cap L \cdot K^{T}$ is $K^{S}$-determinable.

Proof. By definition of Neukirch determinability, $L$ is $K^{S}$ determinable if and only if $\operatorname{Aut}\left(K^{V} \mid K^{S}\right) \cap \operatorname{Aut}\left(K^{V} \mid L\right)$ splits sequence (4.2), which happens if and only if

(i) $\operatorname{Aut}\left(K^{V} \mid K^{S}\right) \cap \operatorname{Aut}\left(K^{V} \mid L\right) \cap \operatorname{Aut}\left(K^{V} \mid L \cdot K^{T}\right)=\{1\}$, and

(ii) $\left[\operatorname{Aut}\left(K^{V} \mid K^{S}\right) \cap \operatorname{Aut}\left(K^{V} \mid L\right)\right] \cdot \operatorname{Aut}\left(K^{V} \mid L \cdot K^{T}\right)=\operatorname{Aut}\left(K^{V} \mid L\right)$.

Condition (i) holds automatically, since $\operatorname{Aut}\left(K^{V} \mid K^{S}\right) \cap \operatorname{Aut}\left(K^{V} \mid K^{T}\right)=\{1\}$; condition (ii) is equivalent to the assertion $L \cdot K^{S} \cap L \cdot K^{T}=L$.

To check that $L \cdot K^{S} \cap L \cdot K^{T}$ is $K^{S}$-determinable, we use the criterion 
just proved: Clearly we need only check that $\left(L \cdot K^{S} \cap L \cdot K^{T}\right) \cdot K^{S} \cap\left(L \cdot K^{S} \cap\right.$ $\left.L \cdot K^{T}\right) \cdot K^{T} \subseteq L \cdot K^{S} \cap L \cdot K^{T}$. We have

$$
\begin{aligned}
&\left(L \cdot K^{S} \cap L \cdot K^{T}\right) \cdot K^{S} \cap\left(L \cdot K^{S} \cap L \cdot K^{T}\right) \cdot K^{T} \\
& \subseteq L \cdot K^{S} \cap L \cdot K^{T} \cdot K^{S} \cap L \cdot K^{S} \cdot K^{T} \cap L \cdot K^{T} \\
&=L \cdot K^{S} \cap L \cdot K^{T}, \text { as required. }
\end{aligned}
$$

Remark (4.4). Proposition (4.3) shows that a $K^{S}$-determinable extension of $K$ is obtainable as the intersection of its inertia field and a Neukirch complement for that inertia field.

Corollary (4.5). Every member of $\left\{K^{V} \mid K^{S}\right\}$ is $K^{S}$-determinable; every member of $\left\{K^{V} \mid K^{T}\right\}$ is $K^{S}$-determinable for all Neukirch complements $K^{S}$.

Proof. $L \supseteq K^{S}$ entails $L \cdot K^{S} \cap L \cdot K^{T}=L \cap L \cdot K^{T}=L$; similarly if $L \supseteq K^{T}$.

Corollary (4.6). For eacb $K^{S}$, the set of $K^{S}$ determinable extensions of $K$ in $K^{V}$ is closed under intersection.

Proof. If $L$ and $M$ are $K^{S}$ determinable, consider $(L \cap M) \cdot K^{S} \cap$ $(L \cap M) \cdot K^{T} \subseteq\left(L \cdot K^{S}\right) \cap\left(M \cdot K^{S}\right) \cap\left(L \cdot K^{T}\right) \cap\left(M \cdot K^{T}\right)=\left(L \cdot K^{S} \cap L \cdot K^{T}\right) \dot{\cap}$ $\left(M \cdot K^{S} \cap M \cdot K^{T}\right)=L \cap M$. The other inclusion is trivial.

Theorem (4.7). A field $L \in\left\{K^{V} \mid K\right\}$ is $K^{S}$ determinable if and only if $\Gamma\left(L \cap K^{S}\right)=\Gamma L$.

Proof. If $L$ is $K^{S}$-determinable, then $\operatorname{Aut}\left(K^{V} \mid L\right)$ is the semidirect product $\operatorname{Aut}\left(K^{V} \mid L \cdot K^{T}\right) \times{ }_{\psi} A u t\left(K^{V} \mid L \cdot K^{S}\right)$; here the action $\psi$ of $\operatorname{Aut}\left(K^{V} \mid L \cdot K^{S}\right)$ on $\operatorname{Aut}\left(K^{V} \mid L \cdot K^{T}\right)$ is induced by inner automorphisms of $\operatorname{Aut}\left(K^{V} \mid L\right)$ on its abelian normal subgroup $\operatorname{Aut}\left(K^{V} \mid L \cdot K^{T}\right)$. The statement $\Gamma\left(L \cap K^{S}\right)=\Gamma L$ is equivalent to $\left(L \cap K^{S}\right) \cdot K^{T}=L \cdot K^{T}$, in turn equivalent to

$$
\operatorname{Aut}\left(K^{V} \mid L\right) \cdot\left[\{1\} \times_{\psi} \text { Aut }\left(K^{V} \mid K^{S}\right) \cap \text { Aut }\left(K^{V} \mid K^{T}\right) \times_{\psi}\{1\}\right]=\operatorname{Aut}\left(K^{V} \mid L \cdot K^{T}\right) \text {. }
$$

Expressing $\operatorname{Aut}\left(K^{V} \mid L\right)$ as the semidirect product which it is in case $L$ is $K^{S}$. determinable, we have

$$
\begin{gathered}
\left(\left[\text { Aut }\left(K^{V} \mid L \cdot K^{T}\right) \times{ }_{\psi} \text { Aut }\left(K^{V} \mid L \cdot K^{S}\right)\right] \cdot\left[\{1\} \times{ }_{\psi} \text { Aut }\left(K^{V} \mid K^{S}\right)\right]\right) \\
\cap\left(\text { Aut }\left(K^{V} \mid K^{T}\right) \times_{\psi}\{1\}\right)=\operatorname{Aut}\left(K^{V} \mid L \cdot K^{T}\right)
\end{gathered}
$$

Conversely, let $\operatorname{Aut}\left(K^{V} \mid L\right)$ be thought of as a subgroup of the semidirect product $\operatorname{Aut}\left(K^{V} \mid K\right)=\operatorname{Aut}\left(K^{V} \mid K^{T}\right) \times_{\psi} \operatorname{Aut}\left(K^{V} \mid K^{S}\right)$. The map $\eta: \tau \mapsto(1, \tau)$ splits 
sequence (4.2) provided $\left\{(\sigma, 1) \mid(\sigma, \tau) \in \operatorname{Aut}\left(K^{V} \mid L\right)\right.$ for some $\left.r \in \operatorname{Aut}\left(K^{V} \mid K^{S}\right)\right\}=$ $\operatorname{Aut}\left(K^{V} \mid L \cdot K^{T}\right)$, yet the former set is exactly $\operatorname{Aut}\left(K^{V} \mid L\right) \cdot \operatorname{Aut}\left(K^{V} \mid K^{S}\right) \cap$ $\operatorname{Aut}\left(K^{V} \mid K^{T}\right)$.

Remark (4.8). For all members $L$ of $\left\{K^{V} \mid K\right\}$, Neukirch-determinable or not, $\zeta\left(L \cap K^{T}\right)=\zeta L$ (see [6]). Theorem (4.7) shows that the Neukirch-determinable extensions of $K$ in $K^{V}$ are exactly those for which the analogous statement holds for value groups.

Corollary (4.9). Every member of $\left\{K^{S} \mid K\right\}$ is $K^{S}$-determinable.

Proof. If $L \subseteq K^{S}$, then $L \cap K^{S}=L$.

Corollary (4.10). All members of $\left\{K^{V} \mid K^{T}\right\}$ and all members of $\left\{K^{T} \mid K\right\}$ are $K^{S}$-determinable.

Proof. By Satz 5 of Neukirch's work [10], the mapping $L \mapsto L \cap K^{S}$ of $\left\{K^{V} \mid K^{T}\right\}$ onto $\left\{K^{S} \mid K\right\}$ preserves value groups. If $L \subseteq K^{T}$ then $\Gamma L=\Gamma K$ yet since $K^{T} \cap K^{S}=K$, we know $L \cap K^{S}=K$.

Corollary (4.11). $L$ is $K^{S}$-determinable if and only if $\left(L \cap K^{S}\right) \cdot\left(L \cap K^{T}\right)=L$.

Proof. Clearly $\left(L \cap K^{S}\right) \cdot\left(L \cap K^{T}\right) \subseteq L$. Further, $\left[\left(L \cap K^{S}\right) \cdot\left(L \cap K^{T}\right)\right] \cap$ $K^{S} \supseteq L \cap K^{S}$, so

$$
\Gamma\left[\left(L \cap K^{S}\right) \cdot\left(L \cap K^{T}\right)\right] \supseteq \Gamma\left(L \cap K^{S}\right),
$$

and $\left[\left(L \cap K^{S}\right) \cdot\left(L \cap K^{T}\right)\right] \cap K^{T} \supseteq L \cap K^{T}$, so

$$
\zeta\left[\left(L \cap K^{S}\right) \cdot\left(L \cap K^{T}\right)\right]=\zeta\left[\left(L \cap K^{S}\right) \cdot\left(L \cap K^{T}\right) \cap K^{T}\right] \supseteq \zeta\left(L \cap K^{T}\right)=\zeta L .
$$

Thus $\Gamma\left(L \cap K^{S}\right) \subseteq \Gamma\left[\left(L \cap K^{S}\right) \cdot\left(L \cap K^{T}\right)\right] \subseteq \Gamma L$ and $\zeta\left[\left(L \cap K^{S}\right) \cdot\left(L \cap K^{T}\right)\right]=$ $\zeta L$. If $L$ is $K^{S}$-determinable, then $\Gamma\left[\left(L \cap K^{S}\right) \cdot\left(L \cap K^{T}\right)\right]=\Gamma L$, and by (22.2) of [4], we have

$$
\begin{aligned}
{\left[L:\left(L \cap K^{S}\right) \cdot\right.} & \left.\left(L \cap K^{T}\right)\right] \\
= & \left(\Gamma L: \Gamma\left(\left(L \cap K^{S}\right) \cdot\left(L \cap K^{T}\right)\right)\right) \quad\left[\zeta L: \zeta\left(L \cap K^{S}\right) \cdot\left(L \cap K^{T}\right)\right]=1 .
\end{aligned}
$$

Conversely, if $L=\left(L \cap K^{S}\right) \cdot\left(L \cap K^{T}\right)$, we have $\Gamma L=\Gamma\left(L \cdot K^{T}\right)=$ $\Gamma\left(\left(L \cap K^{S}\right) \cdot\left(L \cap K^{T}\right) \cdot K^{T}\right)=\Gamma\left(\left(L \cap K^{S}\right) \cdot K^{T}\right)=\Gamma\left(L \cap K^{S}\right)$, so $L$ is $K^{S}$-determinable.

Remark (4.12). Corollary (4.11) shows that a Neukirch-determinable extension is determined by its value group and residue field (hence the name). Indeed, $L \cap K^{S}$ is the unique member of $\left\{K^{S} \mid K\right\}$ having value group $\Gamma L$ and $L \cap K^{T}$ is the unique member of $\left\{K^{T} \mid K\right\}$ having residue field $\zeta L$. In terms of the latticeisomorphisms $v$ and $\zeta$ of Theorem (3.2) and paragraph (2.2), respectively, $L=$ 
$v^{-1}(\Gamma L) \cdot \zeta^{-1}(\zeta L)$. If $L$ is $K^{S}$-determinable, then it is the only $K^{S}$-determinable extension of $K$ in $K^{V}$ having its value group and residue field.

Remark (4.13). For any $L \in\left\{K^{V} \mid K\right\}$, the field $\left(L \cap K^{S}\right) \cdot\left(L \cap K^{T}\right)$ is $K^{S}$. determinable and has value group $\Gamma\left(L \cap K^{S}\right)$ and residue field $\zeta L$; the verification is routine.

Remark (4.14). For any $K^{S}$ and for any prescription $(\Delta, \mathfrak{L})$ of value group and residue field with $\Delta \epsilon\left\{\Gamma K^{V} \mid \Gamma K\right\}$ and $\mathcal{Q} \epsilon\left\{\zeta K^{V} \mid \zeta K\right\}=\{\zeta \Omega \mid \zeta K\}$, there is a $K^{S}$. determinable extension of $K$ in $K^{V}$ having value group $\Delta$ and residue field $\mathcal{L}$ (hence one and only one such field). For, let $L_{1}$ be the unique member of $\left\{K^{S} \mid K\right\}$ having value group $\Delta$, let $L_{2}$ be the unique member of $\left\{K^{T} \mid K\right\}$ having

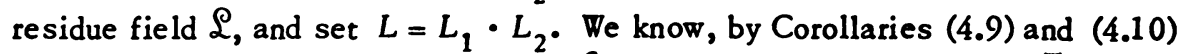
and Corollary (4.15) below, that $L$ is $K^{S}$ determinable. We have $L \cdot K^{T}=$ $L_{1} \cdot L_{2} \cdot K^{T}=L_{1} \cdot K^{T}$ so $\Gamma L=\Gamma L_{1}=\Delta . K^{S}$ determinability implies by Propo-

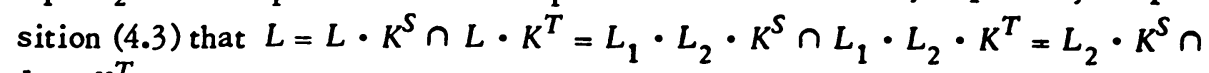
$L_{1} \cdot K^{T}$, so

$$
L \cap K^{T}=L_{2} \cdot K^{S} \cap L_{1} \cdot K^{T} \cap K^{T}=L_{2} \cdot K^{S} \cap K^{T}=L_{2} K^{S} \cap L_{2} K^{T}=L_{2}
$$

since $L_{2}$ is $K^{S}$-determinable. Thus $\zeta L=\zeta\left(L \cap K^{T}\right)=\zeta L_{2}=\mathfrak{L}$.

Corollary (4.15). The set of $K^{S}$-determinable extensions of $K$ in $K^{V}$ forms a sublattice of $\left\{K^{V} \mid K\right\}$.

Proof. We need only show that the set of $K^{S}$-determinable extensions is closed under products. Suppose, then, that $L$ and $M$ are $K^{S}$-determinable, and consider $L \cdot M$. We have $\left(L \cdot M \cap K^{S}\right) \cdot\left(L \cdot M \cap K^{T}\right) \subseteq L \cdot M$, and $\left(L \cdot M \cap K^{S}\right)$ $\cdot\left(L \cdot M \cap K^{T}\right) \supseteq\left(L \cap K^{S}\right) \cdot\left(M \cap K^{S}\right) \cdot\left(L \cap K^{T}\right) \cdot\left(M \cap K^{T}\right)=L \cdot M$ by Corollary (4.11).

Remark (4.16). Proposition (4.3), Corollary (4.5), and Corollary (4.15) show that the lattice of $K^{S}$-determinable extensions of $K$ in $K^{V}$ is precisely the sublattice of $\left\{K^{V} \mid K\right\}$ generated by $\left\{K^{V} \mid K^{S}\right\} \cup\left\{K^{V} \mid K^{T}\right\}$. Corollaries (4.9), (4.10), and (4.11) show that that lattice is identical to the one generated by $\left\{K^{S} \mid K\right\} \cup$ $\left\{K^{T} \mid K\right\}$.

Corollary (4.17). Neukirch's lattice-isomorphisms between $\left\{K^{V} \mid K^{S}\right\}$ and $\left\{K^{T} \mid K^{Z}\right\}$ given by $L \mapsto L \cap K^{T}$ and $M \cdot K^{S} \leftrightarrow M$ preserve residue fields. That is, the place $\zeta$ is a lattice-isomorphism between $\left\{K^{V} \mid K^{S}\right\}$ and $\{\zeta \Omega \mid \zeta K\}$.

Proof. We need only check that the map $M \mapsto M \cdot K^{S}$ of $\left\{K^{T} \mid K^{Z}\right\}$ onto $\left\{K^{V} \mid K^{S}\right\}$ preserves residue fields, as its inverse clearly does. Given $M \epsilon$ $\left\{K^{T} \mid K^{Z}\right\}, M$ is $K^{S}$-determinable by Corollary (4.10), so by Proposition (4.3), $M=$ $M \cdot K^{S} \cap M \cdot K^{T}=M \cdot K^{S} \cap K^{T}$ which has res idue field $\zeta\left(M \cdot K^{S}\right)$. 
Remark (4.18). By Corollary (4.17) we know that for any member $L$ of $\left\{K^{V} \mid K\right\}$, $\zeta\left(\left(L \cap K^{T}\right) \cdot K^{S}\right)=\zeta L$. We remark that, analogous to Theorem (4.7) we have: $L$ is $K^{S}$-determinable if and only if $\zeta\left(L \cdot K^{S}\right)=\zeta L$. For, if $L$ is $K^{S}$ determinable then $\zeta\left(L \cdot K^{S}\right)=\zeta\left(L \cdot K^{S} \cap L^{T}\right)$ by $(2.2)$ and

$$
\zeta\left(L \cdot K^{S} \cap L^{T}\right)=\zeta\left(L \cdot K^{S} \cap L \cdot K^{T}\right)=\zeta L .
$$

Conversely, if $\zeta\left(L \cdot K^{S}\right)=\zeta L$ then $L \cdot K^{S} \cap L \cdot K^{T}=L \cdot K^{S} \cap L^{T}$ which is the unique member of $\left\{L^{T} \mid L\right\}$ having residue field $\zeta\left(L \cdot K^{S}\right)=\zeta L$, so $L \cdot K^{S} \cap$ $L \cdot K^{T}=L$.

Remark (4.19). We observe that for each $K^{S}$, every member $L$ of $\left\{K^{V} \mid K\right\}$ lies between two $K^{S}$-determinable fields which coincide in case $L$ is $K^{S}$-determinable. Namely, $\left(L \cap K^{S}\right) \cdot\left(L \cap K^{T}\right) \subseteq L \subseteq L \cdot K^{S} \cap L \cdot K^{T}$. The smaller field has value group $\Gamma\left(L \cap K^{S}\right)$ and residue field $\zeta L ; L$ is of degree $\left(\Gamma L: \Gamma\left(L \cap K^{S}\right)\right)$ over it. The larger field has value group $\Gamma L$ and residue field $\zeta\left(L \cdot K^{S}\right)$ and is of degree $\left[\zeta\left(L \cdot K^{S}\right): \zeta L\right]$ over $L$.

5. Let $K^{S}$ be a fixed Neukirch complement for $K^{T}$ in $K^{V}$ with Galois group $G^{S}=\operatorname{Aut}\left(K^{V} \mid K^{S}\right)$ and splitting map $\eta: \operatorname{Aut}\left(K^{V} \mid K\right) / \operatorname{Aut}\left(K^{V} \mid K^{T}\right) \rightarrow G^{S}$. We denote $\operatorname{Aut}\left(K^{V} \mid K^{T}\right)$ by $G^{T}$. We regard $A u t\left(K^{V} \mid K\right)$ as the semidirect product $G^{T} \times{ }_{\psi} G^{S}$, where the action $\psi$ of $G^{S}$ on $G^{T}$ is induced by inner automorphisms of $\operatorname{Aut}\left(K^{V} \mid K\right)$ acting on its abelian normal subgroup $G^{T}$.

If $K^{S^{\prime}}$ is another Neukirch complement for $K^{T}$ in $K^{V}$ with splitting map $\eta^{\prime}$, consider $\eta^{\prime}$ as an isomorphism of Aut $\left(K^{V} \mid K\right) / G^{T}$ into $G^{T}{ }_{{ }_{\psi}} G^{S}$. We have, for any $x \in G^{S}, \eta^{\prime}(j x)=\left(\phi_{\eta^{\prime}}(x), x\right)$ for some $\phi_{\eta^{\prime}}(x) \in G^{T}$. Simple computation shows that the map $\phi_{\eta^{\prime}}: G^{S} \rightarrow G^{T}$ is a crossed homomorphism:

$$
\begin{aligned}
\left(\phi_{\eta^{\prime}}(x y), x y\right) & =\eta^{\prime}(j(x y))=\eta^{\prime}(j x) \cdot \eta^{\prime}(j y) \\
& =\left(\phi_{\eta^{\prime}}(x), x\right)\left(\phi_{\eta^{\prime}}(y), y\right)=\left(\phi_{\eta^{\prime}}(x) \cdot\left[\phi_{\eta^{\prime}}(y)\right]^{x}, x y\right) .
\end{aligned}
$$

Here we denote the action $(\psi x)(z)$ by $z^{x}$. If $K^{S^{\prime}}=K^{S}$ then $\phi_{\eta^{\prime}}$ is the trivial crossed homomorphism $\phi_{\eta^{\prime}}(x)=1$ for all $x \in G^{S}$.

It is clear that if $\phi_{\eta^{\prime}}=\phi_{\eta^{\prime \prime}}$ then $\eta^{\prime}=\eta^{\prime \prime}$. Thus the map $\eta^{\prime} \mapsto \phi_{\eta^{\prime}}$ is an injection of the set of splittings of sequence (3.1) into the group of crossed homomorphisms of $G^{S}$ into $G^{T}$.

A principal crossed homomorphism $f$ of $G^{S}$ into $G^{T}$ is by definition one for which $f(x)=\sigma \cdot\left(\sigma^{x}\right)^{-1}$ for some $\sigma \in G^{T}$; we will denote by $f_{\sigma}$ the principal crossed homomorphism determined by $\sigma$.

Lemma (5.1). If $K^{S}$ and $K^{S^{\prime}}$ are $K$-isomorphic, then they are $K^{T}$-isomorphic. 
Proof. Say $K^{S^{\prime}}=\sigma K^{S}$ for some $\sigma \in$ Aut $\left(K^{V} \mid K\right)$. Then $\sigma$ is uniquely expressible as $\tau \cdot \mu$ for some $\tau \in A u t\left(K^{V} \mid K^{T}\right)$ and $\mu \in A u t\left(K^{V} \mid K^{S}\right)$, and we have $K^{S^{\prime}}=$ $\tau \cdot \mu K^{S}=\tau K^{S}$, as $\mu$ leaves $K^{S}$ fixed.

Proposition (5.2). If $K^{S}$ and $K^{S^{\prime}}$, with splitting maps $\eta$ and $\eta^{\prime}$ respectively, are $K$-isomorphic, then $\phi_{\eta^{\prime}} \cdot \phi_{\eta}^{-1}$ is a principal crossed bomomorphism.

Proof. We have $K^{S^{\prime}}=\sigma K^{S}$ for some $\sigma \in \operatorname{Aut}\left(K^{V} \mid K^{T}\right)$ and $\eta^{\prime}(x)=\sigma \cdot \eta(x) \cdot \sigma^{-1}$ for all $x \in \operatorname{Aut}\left(K^{V} \mid K\right) / \operatorname{Aut}\left(K^{V} \mid K^{T}\right)$. Computing $\phi_{\eta^{\prime}}$, we have

$$
\begin{aligned}
\left(\phi_{\eta^{\prime}}(x), x\right) & =\eta^{\prime}(j x)=(\sigma, 1) \cdot(\eta(j(x))) \cdot(\sigma, 1)^{-1}=(\sigma, 1) \cdot\left(\phi_{\eta}(x), x\right) \cdot\left(\sigma^{-1}, 1\right) \\
& =\left(\sigma \cdot \phi_{\eta}(x), x\right) \cdot\left(\sigma^{-1}, 1\right)=\left(\sigma \cdot \phi_{\eta}(x) \cdot\left(\sigma^{-1}\right)^{x}, x\right),
\end{aligned}
$$

so $\phi_{\eta^{\prime}}(x)=\sigma \cdot \phi_{\eta}(x) \cdot\left(\sigma^{-1}\right)^{x}=\sigma \cdot \phi_{\eta}(x) \cdot\left(\sigma^{x}\right)^{-1}=\phi_{\eta}(x) \cdot \sigma \cdot\left(\sigma^{x}\right)^{-1}$ since $\operatorname{Aut}\left(K^{V} \mid K^{T}\right)$ is abelian. Hence $\left(\phi_{\eta^{\prime}} \cdot \phi_{\eta}^{-1}\right)(x)=\sigma \cdot\left(\sigma^{x}\right)^{-1}=f_{\sigma}(x)$, so $\phi_{\eta^{\prime}} \cdot$ $\phi_{\eta}^{-1}=f_{\sigma}$.

As the first cohomology group of $\operatorname{Aut}\left(K^{V} \mid K^{S}\right)$ with coefficients in $\operatorname{Aut}\left(K^{V} \mid K^{T}\right)$ is the quotient of the group of crossed homomorphisms of $\operatorname{Aut}\left(K^{V} \mid K^{S}\right)$ into Aut $\left(K^{V} \mid K^{T}\right)$ by the subgroup of principal crossed homomorphisms (see $[9$, Chapter IV, \$2]), we have

Proposition (5.3). The set of K-isomorphism classes of Neukirch complements for $K^{T}$ in $K^{V}$ is in bijective correspondence with a subset of the group $H_{\psi}^{1}\left(\operatorname{Aut}\left(K^{V} \mid K^{S}\right), \operatorname{Aut}\left(K^{V} \mid K^{T}\right)\right)$ for any fixed Neukircb complement $K^{S}$.

Theorem (5.4). Let $K^{S}$ be a Neukircb complement for $K^{T}$ in $K^{V}$. For a given prescription $(\Delta, \mathfrak{Q})$ of value group extension and residue field extension, with $\Delta \epsilon$ $\left\{\Gamma K^{V} \mid \Gamma K\right\}$ and $£ \epsilon\{\zeta \Omega \mid \zeta K\}$, there are at most $\operatorname{card}\left(H_{\psi}^{1}\left(\operatorname{Aut}\left(K^{V} \mid K^{S}\right)\right.\right.$, Aut $\left.\left.\left(K^{V} \mid K^{T}\right)\right)\right)$ non-K-isomorpbic Neukircb-determinable extensions of $K$ baving the prescribed value group and residue field.

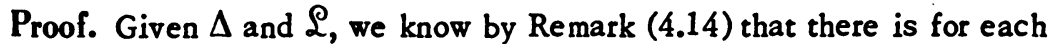
Neukirch complement $K^{S}$ exactly one $K^{S}$-determinable extension of $K$ in $K^{V}$ realizing $(\Delta, \mathfrak{Q})$. We verify that if $K^{S}$ and $K^{S^{\prime}}$ are $K$-isomorphic then so are the extensions $L$ and $L^{\prime}$ which they determine having value group $\Delta$ and residue field $\mathcal{~}$. We assume therefore that $K^{S^{\prime}}=\sigma K^{S}$ for some $\sigma \in$ Aut $\left(K^{V} \mid K\right)$, and as before, may take $\sigma \in \operatorname{Aut}\left(K^{V} \mid K^{T}\right)$. Consider the field $\sigma L$. As $v$ is henselian, $v=v \cdot \sigma$ on any member of $\left\{K^{V} \mid K\right\}$, so $\Gamma(\sigma L)=\Gamma L=\Gamma\left(L \cap K^{S}\right)=\Gamma\left(\sigma\left(L \cap K^{S}\right)\right)=$ $\Gamma\left(\sigma L \cap \sigma K^{S}\right)$, establishing that $\sigma L$ is $\sigma K^{S}$-determinable and has value group $\Gamma L$. Also, the residue field of $\sigma L$ is that of $\sigma L \cap K^{T}$, and as $\sigma$ leaves $K^{T}$ fixed, $\sigma L \cap K^{T}=\sigma L \cap \sigma K^{T}=\sigma\left(L \cap K^{T}\right)=L \cap K^{T}$ which has residue field $\zeta L$. Thus 
$\sigma L$ is $\sigma K^{S}$-determinable and has value group $\Delta$ and residue field $£$, so $\sigma L=L^{\prime}$ by Remark (4.12).

6. Remarks. The author has not succeeded in finding an explicitly non-Neukirch-determinable field. However, since the two extensions $Q_{2}(\sqrt{ } 2)$ and $Q_{2}(\sqrt{ } 6)$ of the field $\mathbf{Q}_{2}$ of 2-adic numbers are non- $\mathbf{Q}_{2}$-isomorphic yet have the same value group and residue field, it is clear that for any Neukirch complement $\mathbf{Q}_{2}^{S}$ for $\mathbf{Q}_{2}^{T}$ in $\mathbf{Q}_{2}^{V}$, not both $\mathbf{Q}_{2}(\sqrt{ } 2)$ and $\mathbf{Q}_{2}(\sqrt{ } 6)$ are $\mathbf{Q}_{2}^{S}$-determinable. Indeed, either one of $\mathbf{Q}_{2}(\sqrt{ } 2), \mathbf{Q}_{2}(\sqrt{ } 6)$ fails entirely to be Neukirch-determinable, or there are non- $\mathbf{Q}_{2}$ is omorphic Neukirch complements for $\mathbf{Q}_{2}^{T}$ in $\mathbf{Q}_{2}^{V}$.

While Neukirch complements are necessarily minimal elements of $\left\{K^{V} \mid K\right\}$ with respect to having value group $\Gamma K^{V}$, the author has been unable to decide whether such a minimal element must be a Neukirch complement. An example of a non-Neukirch-determinable member of $\left\{K^{V} \mid K\right\}$ having value group $\Gamma K^{V}$ would establish the answer negatively. Indeed, if $L \in\left\{K^{V} \mid K\right\}$ has value group $\Gamma K^{V}$, then $L$ contains a minimal element of $\left\{K^{V} \mid K\right\}$ having that property, yet should $L$ contain any Neukirch complement $K^{S}$, then $L$ would be $K^{S}$-determinable, by Corollary (4.5).

\section{BIBLIOGRAPHY}

1. Ron Brown and D. K. Harrison, Tamely ramified extensions of linearly compact fields, J. Algebra 15 (1970), 371-375. MR 41 \#6824.

2. Otto Endler, Über einen Existenzsatz der Bewertungstheorie, Math. Ann. 150 (1963), 54-65. MR $26 \# 3732$.

3. ——, Endliche separable Körpereweiterungen mit vorgeschriebenen Bewertungsfortsetzungen. I, Abh. Math. Sem. Univ. Hamburg 33 (1969), 80-101. MR 39 \#4124.

4. - Valuation theory, Springer-Verlag, Berlin and New York, 1972.

5. H. Hasse, Zwei Existenztheoreme über algebraische Zahlkörper, Math. Ann. 95 (1925), 229-238.

6. L. Hill, Valued fields with prescribed henselizations (to appear).

7. W. Krull, Über einen Existenzsatz der Bewertungstheorie, Abh. Math. Sem. Univ. Hamburg 23 (1959), 29-35. MR 21 \#3406.

8. Saunders Mac Lane and O. F. G. Schilling, A general Kummer theory for function fields, Duke Math. J. 9 (1942), 125-167. MR 3, 266.

9. Saunders Mac Lane, Homology, Die Grundlehren der math. Wissenschaften, Band 114, Academic Press, New York; Springer-Verlag, Berlin, 1963. MR 28 \#122.

10. Jürgen Neukirch, Zür Verzweigungstheorie der Allgemeinen Krullschen Bewertungen, Abh. Math. Sem. Univ. Hamburg 32 (1968), 207-215. MR 38 \#3254.

11. P. Ribenboim, Remarques sur le prolongement des valuations de Krull, Rend. Circ. Mat. Palermo (2) 8 (1959), 152-159. MR 22 \#10985.

12. $\longrightarrow$ An existence theorem for fields with Krull valuations, Trans. Amer. Math. Soc. 105 (1962), 278-294. MR 25 \#5059. 
13. - A short note on Henselian fields, Math. Ann. 173 (1967), 83-88. MR 36 \#144.

14. O. F. G. Schilling, The theory of valuations, Mathematical Surveys, no. 4, Amer. Math. Soc., Providence, R. I., 1950, pp. 70-74. MR 13, 315.

DEPARTMENT OF MATHEMATICS, UNIVERSITY OF COLORADO, BOULDER, COLORADO 80302 